\title{
Sensory theories of developmental dyslexia: three challenges for research
}

Usha Goswami, Centre for Neuroscience in Education, Department of Psychology, Downing Street, Cambridge CB2 3EB, United Kingdom. Email: ucg10@cam.ac.uk

\begin{abstract}
Recent years have seen the publication of a range of new theories suggesting that the basis of dyslexia may be sensory dysfunction. Here, the evidence for and against several prominent sensory theories of dyslexia is closely scrutinized. Contrary to the causal claims being made, my analysis suggests that many proposed sensory deficits may result from the effects of reduced reading experience on the dyslexic brain. I therefore suggest that longitudinal studies of sensory processing, beginning in infancy, are required to successfully identify the neural basis of developmental dyslexia. Such studies could have a powerful impact on remediation.
\end{abstract}

\section{Introduction}

Children with developmental dyslexia fail to acquire efficient reading and spelling skills despite adequate tuition and an absence of overt sensory and/ or neural deficits. Dyslexia is found in all languages so far studied, although its manifestation differs with orthography ${ }^{1}$. Nevertheless, in all orthographies dyslexia disadvantages both school learning and later employment. A U.K. cost-benefit analysis suggested that having dyslexia incurred costs of over $£ 80,000$ on lifetime earnings ${ }^{2}$. Therefore, identifying the underlying cause(s) of dyslexia would enhance the life chances of affected children.

New proposals that suggest that visual and/ or auditory dysfunction underlie dyslexia are driving much current research ${ }^{3}$. However, these theories are challenged by three key facts. First, learning to read itself trains sensory and attentional processes, making it difficult to determine whether sensory deficits are the cause of dyslexia or an effect of the reduced reading experience of individuals with dyslexia. Second, sensory processes underlie all childhood learning. Therefore, evidence of putative sensory deficits that affect only reading is not persuasive. Similarly, a sensory deficit that also occurs in other developmental disorders in which reading is unaffected lacks explanatory power. Third, the protracted developmental timecourse of reading acquisition and its relatively late onset (following tuition from age 5 or later) means that the cognitive effects of sensory dysfunctions should be visible before the onset of reading. Indeed, some of the genes associated with dyslexia are involved in early cortical neuronal migration and are likely to impair sensory processing from infancy onwards $\mathrm{s}^{4,5}$. For example, an important longitudinal study of Norwegian pre-schoolers at family risk for dyslexia found atypical development of sensory structures but not of the temporo-parietal areas usually identified as atypical in neuroimaging studies of older dyslexics, implying that another factor (such as impoverished reading experience) underlies the latter changes ${ }^{6}$. Indeed, if causal to dyslexia, sensory deficits should be detectable even at birth.

In this article, I address these three challenges by scrutinising prominent sensory theories and applying three tests. First, I assess the extent to which developmental research designs relevant to establishing causality have been applied ${ }^{7}$. These are outlined in Box 1, and enable 
the sensory consequences of reduced reading experience to be identified. In general, once the studies that led to the proposal of each theory have been presented, further studies testing these theories that did not use these research designs are omitted from this article (excepting CA-match studies showing that dyslexic performance is unimpaired). Second, I consider the evidence for systematic and hypothesis-driven effects of the proposed sensory deficits on related cognitive skills. Finally, when available, data from longitudinal and infant studies will be discussed to assess evidence for the early fingerprints of sensory dysfunction.

Developmental disorders of learning such as dyslexia represent the extreme bottom end of the normal distribution of a culturally-acquired skill (reading). Pre-literate infants and children and illiterate (unschooled) adults are groups independent of this distribution, and so tests of sensory theories in these populations are of particular importance for identifying causality. It is also important to note that the behavioural manifestations of a sensory impairment may change over the course of development.

\section{Neurotypical reading development}

Learning to read is sometimes erroneously considered a visual skill, but is actually a linguistic process $^{8}$. A brain that can read gains linguistic information from a visual code that represents speech. Hence speech processing skills are integral to reading. Phonological recoding skills (which allow children to convert visual symbols to sound) play a critical early role in accessing meaning from print ${ }^{9}$. These skills develop extremely rapidly in some languages (within the first 3 months of instruction for consistent alphabetic orthographies such as Finnish and Italian $)^{1,9,10}$, and much more slowly in inconsistent alphabetic orthographies (such as English, where whole-word recognition strategies are also useful). Individual differences in 'phonological awareness' - the ability to reflect on the constituent sound elements in words - predicts how well and how rapidly phonological recoding skills are learned in all languages so far studied ${ }^{1,9}$ (Box 2). By contrast, individual differences in visual processing (for example, in visuo-spatial skills) are only occasionally correlated with individual differences in reading acquisition for typically-developing children ${ }^{11,12,13}$.

Cross-language comparisons estimate that between 5 and 6 years of schooling is required for most children to become fluent, silent and efficient readers ${ }^{14}$. After around 10 years of age, cross-language variations in literacy depend on social and economic factors. By contrast, developmental dyslexia occurs at around the same rate $(7 \%)$ across languages ${ }^{2}$, and appears to be unrelated to social or economic factors. Difficulties in the phonological recoding of print to sound lie at the heart of dyslexia in all languages studied ${ }^{1,10}$. A dominant view is that these difficulties arise from impaired or atypical phonological representations of spoken word forms in the neural mental lexicon ${ }^{15,16}$. These atypical representations impair the cognitive achievement of phonological awareness (Box 2). As the acquisition of phonology is multimodal from infancy, with auditory learning supported by visual information from the lips and face and motor information from learning to talk ${ }^{17}$, in principle both visual and auditory sensory dysfunction could cause the cross-language 'phonological deficit' that characterises developmental dyslexia.

Given the core developmental role of phonological recoding, however, a child that is reading less text will quickly accumulate far less experience of the component skills of reading. For example, visual scanning, oculomotor control and associated visuo-spatial attention skills will 
all be practiced less, as will phonological recoding itself. Such a child would quickly begin to show apparently atypical sensory processing. These interpretive issues are magnified when dyslexic adults are studied instead of children. By adulthood the dyslexic brain has had 15 years or more of impoverished reading experience. In the cognitive domain it is wellestablished that the "rich get richer" during the development of reading (for example, children who read more develop better vocabularies, which in turn enhances their phonological recoding skills) ${ }^{18}$. These effects also apply to sensory processing, compounding the need for sensory dysfunction theories rigorously to test causality. Furthermore, the effects of reduced reading experience may be expected to vary across languages. For example, a child reading a transparent script like Italian, in which letter-by-letter recoding to sound is a successful early strategy, would experience far more spatial serial processing of letters than a child reading an opaque script like English. Therefore, a sensory dysfunction that causes dyslexia should be found across languages, and its effects should vary in systematic ways according to variations in orthography and phonology.

\section{Impairments in visual processing}

\section{Magnocelluar theory}

When visual information leaves the occipital lobe, it follows two main pathways. The ventral pathway is involved in object identification and recognition, whereas the dorsal pathway primarily processes spatial position. The dorsal pathway develops more slowly ${ }^{19}$, and encompasses the subcortical magnocellular system, which detects motion, low-frequency spatial information and high-frequency (fast) temporal information: information critical for representing and parsing the visual field. The magnocellular theory of dyslexia states that deficits in magnocellular visual processing are a primary cause of the disorder ${ }^{20}$.

The magnocellular theory developed from psychophysical and post-mortem data suggesting abnormal function and appearance of magnocells in the lateral geniculate nucleus in individuals with dyslexia ${ }^{21,22}$. Importantly, these early data relied on chronological age (CA) match studies, in which dyslexic readers were matched to good readers of the same age. As reading experience differs dramatically in CA match designs, the results of these studies are completely ambiguous with respect to causality. The magnocellular deficit was reported to extend to dorsal cortical systems in a further series of CA-matched studies that revealed dyslexic deficits in perceiving coherent motion in random dot arrays, in binocular control, and (in fewer studies) in other "magnocellular" tasks such as the frequency doubling illusion $^{23,24,25}$. These studies led to the proposal that difficulties in vergence control (directing both eyes to the same point in space during the sequential tracking of print) led to 'reversed' letters effects when reading (reading WAS for SAW, for example). According to this proposal, difficulties in guiding eye movements caused unstable or jumbled visual representations of words and hence reading difficulties. Although all children go through a developmental stage of letter reversal during learning to read $^{26}$, imaging research revealed atypical (reduced) neural activity in the brain area related to motion processing (area V5/MT, visual area 5 in extrastriate visual cortex, also known as middle temporal area) in children and adults with dyslexia ${ }^{27}$. This was taken as further evidence for the theory ${ }^{20}$, even though CA-match designs were used and cause and effect cannot be disentangled. 
There are few reading level (RL)-match or longitudinal studies of magnocellular processing, and few cross-language or intervention studies. Only one RL-match study has reported a positive result, based on the frequency doubling illusion ${ }^{28}$. This Italian study found that dyslexic children had higher thresholds in the illusion than both CA and RL controls ${ }^{28}$. By contrast, a CA-match German study measured vergence control and sequential tracking directly by asking children to identify identical letters in meaningless letter strings ${ }^{29}$. Dyslexic children were as good as the CA-matched controls and showed equivalent eye movement patterns. Furthermore, their ability to perceive coherent motion was not related to their performance. A Dutch longitudinal study of pre-reading children at familial risk for dyslexia, however, reported a significant relationship between coherent motion detection and reading one year later in control children ${ }^{30}$. By contrast, a study of English-speaking preschool children (not dyslexic) found no relationship between later reading and performance on the coherent motion task ${ }^{25}$. Instead, individual differences in normative performance with the frequency-doubling illusion were related to later reading skills.

More systematic application of the research designs identified in Box 1 in more languages may clarify this currently mixed picture. It is also important that studies include, as a control, parvocellular tasks that are of equal difficulty to the magnocellular tasks: some data show that once task difficulty is equated, participants with dyslexia are impaired in both types of task, weakening claims that magnocellular dysfunction has a causal $\mathrm{effect}^{31}$.

The results of an important study that investigated the effects of training phonological recoding skills pose further difficulties for the magnocellular hypothesis ${ }^{32}$. This study used neural imaging to measure activity in V5 prior to and following training of dyslexic children, and made comparisons with CA- and RL-matched controls (Figure 1). Training consisted of tutoring programmes that taught the component skills of either reading (such as phonological skills) or mathematics. Before training, children with dyslexia showed reduced V5 activation compared to CA-matched children, but equivalent activation compared to RL-matched children. Following phonological training, which improved reading skills in the dyslexic group, V5 activity in the dyslexic group increased. Importantly, mathematics training did not improve reading skills or V5 activity, suggesting that the relationship between magnocellular function and reading is mediated by reading experience ${ }^{32}$. Sensory processes such as oculomotor control, visual attention and spatial position encoding are all trained when print is recoded to sound, and all these processes are subserved by the magnocellular visual system.

Currently no studies have examined systematic effects on other cognitive systems that depend on magnocellular function. One obvious prediction is that spatial orienting in infancy should be affected ${ }^{33}$. Additional longitudinal studies are required, examining magnocellular function in infants and pre-readers at family risk for dyslexia. Studies of magnocellular function in illiterate adults are also absent, as are studies utilising Box 1 research designs with readers of non-alphabetic scripts. As the grain size (the unit size at which spelling maps to phonology) of the visual code is usually larger in non-alphabetic scripts, processes such as fine ocularmotor control may receive less training via reading experience in these scripts. Hence it may be possible to demonstrate parametric effects of orthography on V5 activation (that is, dyslexic readers of languages like Chinese may show smaller V5 effects). Along these lines, a study in Hebrew found no magnocellular deficits in dyslexia in a series of perceptual tasks with adults in a CA-match study ${ }^{34}$. 
Despite its prominence, therefore, the magnocellular theory appears to identify a sensory dysfunction that is primarily related to reduced reading experience. In addition, immaturity of the dorsal visual system appears to characterise a number of developmental disorders, including dyslexia, autism, Williams syndrome and dyscalculia ${ }^{35}$. Dorsal deficits are hence not specific to dyslexia, further weakening the causal claims of the magnocellular theory.

\section{Impairments in visual attention.}

The visual environment presents far more information than can be processed effectively. Visual attention enables the selection for processing of information most relevant to ongoing behaviour, and the suppression of information that is less relevant (inhibition). Two prominent theories suggest that impairments in visual attention may underlie dyslexia.

\section{Impaired visual attention span.}

The span of visual attention is the amount of visual information that can be maintained across brief disruptions to sensory input (for example, during blinking or saccades). Visual span is vital for coherent experience. One theory suggests that visual span capacity is reduced in developmental dyslexia and that this primary impairment in the ability to process multipleitem arrays limits reading development ${ }^{36,37,38}$.

Visual attention span is typically measured as the number of individual elements that can be processed simultaneously in the "attentional window", the region in visual space to which selective attention is being directed. A classic span task presents an array of 5 elements (such as 5 letters or digits) very briefly, and then records reaction time when naming either all the elements in the array (full report) or single elements at different cued positions (partial report). Usually reaction times show an M-shape (faster report for the elements in positions 1, 3 and 5) and accuracy shows a W-shape (greater accuracy at these positions). Reduced speed and accuracy at positions 2 and 4 is argued by some to reflect crowding effects - visual interference from the elements flanking the target. Crowding effects can be reduced by surrounding the target with congruent stimuli that share its global contours (lines, curves etc) - producing congruence effects.

Crowding effects appear to be larger in Italian dyslexic children than in controls in CA-match designs $^{39,40}$ and CA-match visual span studies with French-speaking children show a reduced visual span in children with dyslexia ${ }^{36,37}$. Furthermore, one French RL-match study found that severely impaired older dyslexic children (aged $9-14$ years with an average reading age of 7 years) showed poorer phonological recoding skills and visual spans than RL-matched controls (aged 8 years) ${ }^{38}$. As outlined above, such positive results in CA-match designs could reflect reading experience. In addition, the classic visual span task uses letters, which are known to be processed less efficiently by children with dyslexia ${ }^{41,42,43}$ and the oral report method requires rapid access to phonology, also impaired in dyslexia. Hence any visual attention task based on letters or requiring oral reporting is inherently ambiguous with respect to causality ${ }^{41}$.

When the research designs summarised in Box 1 are applied to visual span theory, the confounding role of reading experience becomes apparent. For example, a recent Portuguese study compared illiterate adults with dyslexic children and typically-reading (RL- and CA- 
matched) controls in a visual attention task measuring congruence effects ${ }^{44}$. Both letter stimuli and pseudo-letter stimuli were presented. All groups showed congruence effects for pseudo-letter stimuli; however, no congruence effect was found for the illiterate adults when letter stimuli were used. A significant congruence effect was found for the dyslexic children using letter stimuli. This result suggests that reading experience governs the emergence of congruence effects for letters. Indeed, the strongest predictor of the size of the congruence effect for letter stimuli in dyslexic children was phonological recoding ability ${ }^{44}$.

As described above, it is important to determine whether there are systematic effects of a proposed sensory deficit on stimuli that are not letters or words. The classic 5-element visual span task was used in two studies ${ }^{41,45}$ that compared visual span for arrays of letters and digits with visual span for non-alphanumeric stimuli (Figure 2). In a CA-match design, children with dyslexia showed reduced visual spans for letters and digits. However, their performance was identical to that of CA-matched controls for unfamiliar symbols ${ }^{41}$ and coloured dots ${ }^{45}$. Hence despite their reading difficulty and reduced reading experience, French dyslexic children showed preserved visual spans for materials that had not been recoded to sound with the same frequency as letters and digits. Again, this supports a non-causal interpretation of visual span deficits. Currently lacking are training studies or longitudinal studies with at-risk infants or pre-readers (Box 1).

It is important to note that even if reduced visual attention span, increased crowding effects and letter congruence effects are a product of reading experience, dyslexic reading may be facilitated if these visual factors are minimised. Increased spacing between letters, hypothesised to reduce crowding, significantly improved sentence reading accuracy for 10year-old French and Italian dyslexic children ${ }^{46}$. However, an Italian RL-matched control group did not show accuracy benefits from increased spacing (no French RL group was included). This suggests that increased spacing, a typical feature of books for younger readers, has a selective benefit in dyslexia. Replication of this finding in other languages is required: more complex orthographies such as English, that require orthographic processing at larger grain sizes, may not show equivalent between-letter spacing effects.

\section{Impaired visuo-spatial attention.}

A second visual attention theory argues that a 'spatial cueing' deficit (impaired ability to orient spatial attention) causes dyslexia ${ }^{47,48,49}$. An efficient attentional system can orient rapidly in response to exogenous cues ${ }^{50}$. Exogenous cues are independent of the information being attended to (such as letter colour when reading), but have an automatic sensory "cueing" effect, facilitating stimulus detection and response time by orienting attention to the most informative aspects of the visual field without moving the eyes. According to visuospatial attention theory, attentional shifting is "sluggish" in individuals with dyslexia". It is suggested that the dyslexic brain cannot move smoothly from letter to letter while suppressing flanking letters when recoding print to sound.

Even infants show visual cueing effects ${ }^{50}$. When visual array sizes exceed visual span capacity, infants as young as 5 months show processing benefits from exogenous cues. However, attentional cueing is not an all-or-none process even in infancy, and experience of cues with different perceptual characteristics (motion versus a stationary square, for example) is required for attentional facilitation. Hence it is important to establish that reading 
experience per se is not facilitating typically-developing children's use of spatial cues in the paradigms used to establish the dyslexic 'spatial cueing' deficit.

The classic spatial cueing paradigm compares the effects of "valid" (informative) and "invalid" (uninformative) cues. These cues are usually presented with different time delays between cue and target presentation. A "cueing effect" results in enhanced orienting (measured via faster RTs or better accuracy) to the target. A series of studies with Italian dyslexic children has shown impaired spatial orienting of attention ${ }^{48,49,52,53}$ (Figure 3). Importantly, however, only Italian dyslexic children with phonological recoding deficits showed a spatial cueing deficit ${ }^{48,49}$.

One strength of the Italian studies (Figure 3) has been the inclusion of RL-matched controls and the use of longitudinal designs (Box 1). For example, a key study involving 22 10-yearold dyslexic children showed no spatial cueing effect at $100 \mathrm{~ms}$ delay in 13 dyslexic children with poor phonological recoding, who instead showed a (sluggish) cueing effect at $250 \mathrm{~ms}^{49}$. Younger RL-match children in the study could utilise the faster (100ms delay) cue. In a longitudinal study, 82 Italian pre-readers were studied ${ }^{52}$. Of these, 14 children were later classified as poor readers and were found to have had reduced pre-reading attentional orienting. However, the poor readers had also had poorer pre-reading phonological awareness compared to the 68 unimpaired readers, and verbal IQ was not controlled between groups, complicating interpretation. Thus, although a number of the research designs described in Box 1 have been utilised to explore the role of sluggish orienting of visuo-spatial attention in dyslexia, the causal claim currently depends on a mixture of effects on accuracy and RT and there is not clear evidence for sluggish attention in every study.

A recent training study complicates the picture further. Twenty 10-year-old Italian dyslexic children were given experience with either active or non-active video games ${ }^{53}$ over 9 days of training. The active gaming produced significant gains in visual spatial attention and reading speed (although not reading accuracy). However, the active gamers also improved their speed of phonological recoding to sound (in a pseudoword reading measure). Hence, the study did not establish that the video gaming improved spatial attention and therefore improved word reading speed. The reading speed improvements could equally have been caused by the increased speed of phonological recoding found for the active gamers.

The fact that only Italian dyslexic children with phonological recoding problems have been shown to exhibit a spatial cueing deficit may again point to reduced reading experience as the driver of this attentional deficit. Italian dyslexic children who can read pseudowords efficiently do not show spatial cueing problems (they are also unimpaired in the magnocellular frequency doubling paradigm ${ }^{28}$ ). This group of children are seldom described in any detail, but their lack of visual attention impairments critically undermines the causal argument. These children show impaired real word and text reading with unimpaired pseudoword reading, and comprise around half of the dyslexic sample in the Italian studies $^{48,49}$. The attentional deficit in Italian is also limited to impaired inhibition (suppression) in the right visual field (RVF) in some studies ${ }^{47,48}$. This again suggests a role for reduced experience of recoding print to sound. The reduced practice in serial left-to-right focussing of spatial attention that accompanies reduced experience of recoding print to sound should selectively affect the right attentional inhibition system ${ }^{48}$. One important test would be to study an orthography such as Hebrew, in which orthographic processing is right-to-left. If 
reduced practice in phonological recoding underlies spatial cueing deficits in dyslexia, Hebrew readers with dyslexia should show impaired inhibition in the left visual field.

Italian is one of the most consistent alphabetic orthographies, and recoding individual letters to sound is the hallmark of early Italian reading. This linguistic factor could also explain why such strong spatial cueing deficits are found in Italian dyslexic children ${ }^{49}$. German is also a relatively transparent language, but is less transparent than Italian. German dyslexic children showed no spatial attention deficit compared to CA controls and no RVF effect in a spatial attention task requiring precedence detection ${ }^{54}$.

Currently, there are no studies of infants or of children at family risk for dyslexia that show spatial cueing deficits prior to learning to read. Similarly, there are no studies exploring other likely consequences of a spatial cueing deficit, such as reduced orienting of spatial attention in the natural visual environment. Specification of the temporal parameters governing "sluggishness" across different paradigms/age groups is also required, to give in principle explanations for the range of cue-target delays utilised to date. Finally, languages other than Italian also need to be tested.

Currently, the simplest explanation of the data is that reduced experience of phonological recoding, which for Italian children does involve moving spatial attention from letter to letter, underlies the spatial cueing deficit in dyslexia. Such fine-grained sequential shifts of attention are practiced far less when reading scripts such as Chinese, or even when reading inconsistent alphabetic scripts such as English. It is possible that more systematic cross-language investigations would reveal parametric effects of orthography, with the visuo-spatial attention deficit actually driven by reduced reading experience.

Finally, it should be noted that magnocellular and visual attention theories are now merging, with impaired (magnocellular-dependent) spatio-temporal parsing of the visual text (a process linked to parietal cortex) claimed as a sufficient cause for dyslexia ${ }^{55}$. However, this 'metatheory' currently lacks any systematic application of the research designs in Box 1.

\section{Impaired auditory processing}

As speech is an acoustic signal, auditory dysfunction offers a parsimonious developmental cause of the phonological deficits that characterise dyslexia. However, the speech signal is very complex, and our understanding of the neural processing of speech is incomplete. Nevertheless, sensitivity to rhythmic information in speech is present in the womb, neonates can distinguish languages that are members of different rhythm classes ${ }^{56}$, and sensitivity to phonetic information is present soon after birth ${ }^{17}$. Furthermore, the cortical oscillatory mechanisms (Box 2) underpinning speech encoding seem to function at a range of temporal rates in the womb and at birth ${ }^{57}$. The perceptual organization of speech information (assigning acoustic elements to the groupings comprising linguistic units, see Box 2) takes longer, and also benefits from top-down learning ${ }^{58}$. Impairments in the ability to utilise the acoustic structure of the speech stream should have consequences for phonological processing.

\section{Rapid auditory processing (RAP) deficit.}


The first auditory theory of sensory dysfunction proposed developmental difficulties in processing auditory information that arrived rapidly and sequentially ${ }^{59}$. RAP theory focused on spectral structure (the variations in frequency that occur as a speaker moves from consonant to vowel, for example), as the spectral changes related to linguistic units are typically rapid (within $40 \mathrm{~ms}$ for formant transitions ${ }^{58}$ ). Work with children with specific language impairment (SLI) ${ }^{60,61}$ demonstrated that their ability to process rapidly-arriving (within $\sim 40 \mathrm{~ms}$ time window ${ }^{59}$ ) auditory information was impaired compared to that of CAmatched control children. In a subsequent CA-match study, RAP problems were demonstrated in 8 of 20 dyslexic children tested ${ }^{62}$, leading to the claim that difficulties in processing rapidly-changing information in speech (such as formant transitions) caused the phonological deficit in developmental dyslexia ${ }^{59}$ (Figure 4a). However, the reliance on CAmatch data meant that this proposal generated considerable debate, with critics failing to find a RAP deficit in dyslexia ${ }^{63,64}$, showing that comparable difficulties were not apparent with speech stimuli ${ }^{65}$, or showing that slowing down temporal information in speech did not improve the phonological performance of children with dyslexia ${ }^{66}$.

Subsequent RAP studies have continued to utilise ambiguous CA-match designs; however, training studies and longitudinal studies are also available. A computer application intended to train RAP has shown apparent within-participant benefits ${ }^{67,68}$ (although no control groups of children who play similar acoustic software games that train non-rapid auditory processing have been studied). These software games also explicitly train phonological awareness, hence the gains that have been demonstrated are inherently ambiguous. In a longitudinal study, American pre-readers at familial risk for dyslexia showed differences compared to typicallydeveloping CA controls in brain activity (specifically, hypoactivation in left frontal cortex) when listening to non-speech stimuli with rapid frequency transitions ${ }^{69}$. As similar hypoactivation characterises older children and adults with dyslexia in RAP tasks, the atypical activation demonstrated was argued to be supportive of RAP theory ${ }^{69}$.

Important longitudinal work with English-learning infants may be promising in terms of demonstrating causality; nevertheless, results to date implicate a role for RAP deficits in SLI and not dyslexia ${ }^{70}$. Furthermore, longitudinal studies of RAP in preschoolers in other languages show negative results. For example, Dutch pre-schoolers at familial risk of dyslexia failed to show any RAP difficulties, and performance in RAP tasks did not predict later phonological awareness ${ }^{71}$. The claim that RAP deficits cause dyslexia is also undermined by a recent CA-match study that found that children with dyslexia were significantly better than CA-matched controls in discriminating rapid rises in frequency that changed a synthesised BA syllable into the syllable $\mathrm{WA}^{72}$. The dyslexic group were able to discriminate between BA and WA syllables distinguished by a $15 \mathrm{~ms}$ rise in frequency, whereas CA-matched controls required frequency rises of $30 \mathrm{~ms}$ or more. If replicated, such a result would appear to rule out formant transitions as a source of the phonological deficit in dyslexia.

Meanwhile, as phoneme awareness is largely a product of learning an alphabetic script (Box 2), experience of phonological recoding is vital for learning phonemic structure from alphabetic information. Difficulties in perceiving rapid information in speech should hinder children's ability to learn phonemic structure, and again the severity of impairments should vary with orthographic consistency. Accordingly, studies of French dyslexic adults showing atypical neural processing of amplitude modulations in the gamma range ${ }^{73}$ are not evidence 
that this atypical processing causes dyslexia. The atypical processing could equally be a result of years of reduced phonological recoding experience. Similarly, if Dutch adults with dyslexia show comparable neural processing (similar multi-voxel activity) to age-matched controls in a syllable discrimination task, this does not prove that dyslexia arises from an impairment in accessing intact phonological representations ${ }^{74}$. Dutch is another transparent orthography, so even reduced experience of phonological recoding to sound would have developmental effects on the quality of phonological representations.

Finally, there are currently no studies exploring whether musical cognition is impaired as a result of putative RAP difficulties. Logically, it should be affected. However, developmental studies of categorical perception using synthetic speech stimuli have reported significantly more sensitive performance by dyslexic children ${ }^{75,76}$. In categorical perception tasks dyslexic children show better discrimination within a phonemic category than both CA- and RLmatched controls ${ }^{76}$. These data have been interpreted as showing that allophonic perception, normally discarded in infancy ${ }^{17}$, is preserved in dyslexia ${ }^{75}$. Although this position is the theoretical opposite of RAP theory, it is important to note that significant dyslexic advantages in allophonic perception would be consistent with demonstrations of atypical neural activation in RAP studies ${ }^{68,69}$. However, the atypical activation would reflect allophonic processing. Currently, therefore, the evidence for the RAP theory of developmental dyslexia does not meet the conditions for establishing causality.

\section{Amplitude modulation (rise time) deficit}

A more recent theory concerning auditory temporal processing focuses on relatively slow information and concerns intensity (amplitude) rather than frequency (pitch). Rise time theory proposes that there are dyslexic impairments in discriminating amplitude envelope rise times at slower temporal rates, which affect the detection of speech rhythm and prosody ${ }^{77,78}$. The amplitude envelope of speech is its slow-varying energy contour and contains a range of amplitude modulation patterns at different temporal rates. These rates have different 'rise times' (the time required to reach the modulation peak). Recent modelling of the patterns of amplitude modulation at different frequencies and timescales in child-directed speech suggests that these slower AM patterns support the developmental emergence of phonological awareness at the larger grain sizes that are available to pre-literate children $^{79,80,81}$ (Figure 4 and Box 2). The three dominant rates of AM in child-directed speech $^{79}$ are shown in Figure $4 b$.

Rise time is related to the perceptual organisation of speech rhythm and syllable structure, and school-age children do not yet show adult-like use of the AM structure of $\operatorname{speech}^{58}$. However, a series of studies measuring rise time sensitivity in dyslexic children across languages ${ }^{82,83,84,85,86}$ all reported impaired discrimination of rise time compared to CA controls, with dyslexic children performing like younger RL-matched children. Only Greek dyslexic children showed no differences compared to either CA-match or RL-match groups, an ambiguous result ${ }^{87}$. Significant relationships between phonological awareness and rise time sensitivity were found in all studies reporting positive results. These early studies all explored rise time relationships with sub-syllabic phonological awareness (onset-rime and phoneme awareness). 
Deficits in novel tasks that theoretically should be affected by impaired rise time discrimination were subsequently demonstrated for English children with dyslexia. These children showed previously unsuspected prosodic deficits, with younger dyslexics (9-yearolds) showing significantly poorer prosodic awareness than RL controls (7-year-olds) ${ }^{88,89}$. Rhythmic processing of non-speech sounds was also affected, with dyslexic children showing impaired tapping to a metronome beat at $2 \mathrm{~Hz}$, and individual differences in tapping accuracy that were related to phonological awareness and reading ${ }^{90}$. Most strikingly, children with dyslexia were also significantly poorer than younger RL-matched children at judging rhythm in music ${ }^{91}$. In fact, the musical rhythm task was a stronger predictor of reading than phonological awareness in this study ${ }^{92}$. Furthermore, in a 7-year longitudinal study following around 40 children with dyslexia along with CA- and RL-matched controls from the age of 8 years, a significant dyslexic impairment in rise time sensitivity compared to RL-matched children emerged at the age of 12 years $^{91}$ (by that test point, RL controls were aged 10 years). This makes it unlikely that the rise time impairment in dyslexia is a sensory product of reduced reading experience. Superior metacognitive skills may have masked this sensory difference at earlier test points.

In a different longitudinal study, rise time sensitivity in English-speaking pre-readers (4-yearolds) was a significant predictor of rhyme awareness at age $5^{93}$. The longitudinal study of atrisk Dutch pre-readers mentioned above found that sensitivity to slow frequency modulations predicted later reading ability and phonological awareness ${ }^{30}$.

Many of the conditions for establishing causality have therefore been met for rise time theory, although further longitudinal studies are required. To date, there have been 2 major longitudinal studies following babies at family risk for dyslexia from birth, one Finnish and one Dutch. Neither study included rise time measures; nevertheless, both identified a range of neonate and infant auditory weaknesses using EEG that predicted later phonological awareness and reading ability ${ }^{94,95,96}$. A small-scale family-risk longitudinal study in English revealed timing difficulties in syllable production for the at-risk children at ages 2 and $3^{97}$.

The results of training studies also support rise time theory. Training studies with both dyslexic and poor readers have shown that behavioural interventions designed to enhance the perception of rhythm in language (using music, drumming, marching and poetry) improved phonological awareness, reading and spelling ${ }^{98,99}$. Motor entrainment (assessed using a tapping-to-music task) was also measured and improvements in entrainment were significantly related to reading improvements ${ }^{99}$. The role of entrainment to the beat may be important conceptually in explaining neurocognitive links between rise time, rhythm, phonological awareness and reading ability.

These potential neurocognitive links were spelled out explicitly in temporal sampling (TS) theory, which was developed to take account of the auditory cortical oscillatory hierarchy shown in Box $2^{78}$. TS theory proposed that the ability of dyslexic children to perceive the patterns of amplitude modulation at slow timescales in speech might be impaired by poor rise time detection (Figure 4b). Rise times function as auditory "edges", resetting ongoing neuronal oscillations so that oscillatory peaks are aligned with AM peaks. Atypical oscillatory alignment would thus affect the perceptual organisation of AMs, meaning that stressed syllables, syllables and the onset-rime division would be poorly encoded. Such AM difficulties would affect phonological skills across languages. If the higher levels of the 
oscillatory hierarchy entrain poorly to AM information, this would also affect the downstream identification of smaller units such as phonemes when reading is taught (Box 2).

Neural tracking of rise times and the amplitude envelope can be measured directly using EEG. Hypothesis-driven EEG studies revealed that dyslexic children indeed exhibited different ERPs to amplitude envelopes with longer (90ms) rather than shorter (15ms) rise times compared to CA controls ${ }^{100}$. The same children were significantly impaired in neuronal entrainment to a rhythmic syllable stream presented at a $2 \mathrm{~Hz}$ rate ${ }^{101}$. When hearing or seeing a speaker rhythmically repeating "ba, ba, ba..", the children with dyslexia showed phase alignment at less informative temporal points in the incoming signal, which degraded speech representational quality ${ }^{101}$. The difference in preferred delta phase between the groups was 0.16 radians at $2 \mathrm{~Hz}$, which is the equivalent of $12.8 \mathrm{~ms}$, an interval within the phoneticallyimportant $40 \mathrm{~ms}$ window identified earlier by RAP theory ${ }^{59}$. Therefore, the consistent timing difference could have cascading consequences for the optimal encoding of phonetic information by these children. Individual differences in preferred phase were indeed related to performance in a phoneme deletion task ${ }^{101}$. Importantly, different phase alignment between groups was not found in a visual speech control condition ${ }^{101}$. Nevertheless, RL-match EEG studies are currently missing.

Also missing is a demonstration that neural encoding of low-frequency envelopes is impaired when children are listening to sentences. Studies of illiterate adults would also be interesting. Illiterate adults can detect rhymes but not phonemes ${ }^{102}$, which would suggest that rise time sensitivity should be unimpaired in these individuals. Further cross-language studies are also required as rise time theory would predict prosodic and rhythmic difficulties in dyslexia across languages. If difficulties with syllable stress were found in Italian dyslexic children who do not show phonological recoding difficulties (as well as in those who do), this would be particularly persuasive evidence for a primary auditory deficit. Difficulties with syllable stress and prosodic awareness would also be predicted for Greek dyslexic children.

\section{Future Directions}

This necessarily selective review of studies of sensory dysfunction in children with dyslexia suggests that differences in reading experience (phonological recoding) drive the visual sensory processing difficulties that have been identified in individuals with dyslexia. It is too early to dismiss the visual theories, however, as the available studies suffer from under-use of the research designs described in Box 1. There is also little data on the typical development of vergence control and visual attention, and no data on vergence control and visual attention in illiterate populations. Furthermore, ameliorating visual difficulties by increasing letter spacing or using shorter lines of text seems to have a positive impact on reading by children and adults with dyslexia ${ }^{46,103}$.

Based on current data, temporal sampling theory ${ }^{78}$ comes closest to meeting the conditions for establishing causality. Rise time theory can also explain why deficient phonemic processing and atypical rapid-rate acoustic processing may occur in dyslexia. This would be a likely consequence of downstream perturbation of the acoustic modulation hierarchy in speech (Box 2). Low-frequency AM is detected by the foetus. If extraction of primitive phonological structure (the nested hierarchy of prosodic stress, syllables and onset-rime units, 
Box 2) is mediated by neuronal oscillatory entrainment at different speech timescales, and if entrainment to slower AM rates is impaired in developmental dyslexia, then the mental lexicon would develop a different 'auditory organization, ${ }^{104}$ from birth. Phonological representations would develop differently in dyslexia: for example, impaired speech rhythm detection might be compensated by extra sensitivity to phonetic cues ${ }^{78}$. Developmentally, this atypical trajectory would preserve spoken language processing while impairing written language processing (for example, too many 'phonemes' would map to one letter) ${ }^{75,76}$.

It is also possible that a primary deficit in a third factor, such as pan-sensory oscillatory entrainment, could explain both the auditory and visual sensory difficulties documented here $^{3,105,106,107}$. As amplitude envelope rise time relates to signal intensity, it may be that oscillatory entrainment to luminance or spatial rather than temporal information is impaired in the dyslexic visual system. Systematic studies are required across languages to explore auditory, visual and motor processes. It is important to note also that atypical sensory processing from infancy would lead to developmental compensation by other sensory processes and/or systems, and this too could be tested in hypothesis-driven ways ${ }^{105}$. A useful way forward experimentally would be for all the key tasks identified in this review to be included in longitudinal studies, beginning in infancy, in as many languages as possible. Identifying the correct cause/s of dyslexia would benefit the education of millions of children, enabling early environmental enrichment. In the future, accurately-targeted enrichment may even allow this academically-limiting disorder of learning to be eradicated. 


\section{Box 1}

\section{Research designs that can disentangle cause from effect in dyslexia research}

The reduction in reading experience that is inherent in being dyslexic can itself cause differences in sensory processing between dyslexic and control participants. Adoption of the following research designs can control to some extent for the effects of reading experience on the brain. If similar outcomes are found using combinations of these designs, causality is more likely to be present.

- Reading level (RL) match design. In these studies, children with dyslexia (10-yearolds, for example) are matched to younger children (such as 7-year-olds) who have attained the same level of reading. If dyslexic children perform worse than the younger RL-matched children this suggests a causal role for the factor being investigated, as the dyslexic children have higher chronological and mental ages and better metacognitive skills. Subsequent longitudinal and training studies are required to establish causality. A result in which both groups perform equally is causally ambiguous. The higher metacognitive abilities of the dyslexic children may be masking a deficit.

- Research with illiterate adults. Illiterate adults have never been taught to read and hence have never developed a specialised letter processing system. Nevertheless, they have grown up in cultures in which letters and print are ubiquitous. If illiterate adults show no deficit in a sensory task involving letters and words in which dyslexic children perform worse than age-matched controls, this is a good indication that the sensory deficit in the dyslexic children is a product of reduced reading experience.

- Research with pre-readers. Infants and pre-reading children provide a critical test for causal sensory theories of dyslexia. The candidate mechanism should already show impairments in pre-readers who go on to be diagnosed with dyslexia, and should systematically affect reading acquisition in these children once instruction commences.

- Longitudinal studies. The best studies, but also the rarest, follow the same children over the whole learning trajectory, to establish temporal cause versus effect.

- Training studies. The strongest test of causation in development is an intervention study. If sensory process A causes cognitive deficit B, then providing training in A should ameliorate B. A control group should receive matched training omitting the key sensory variable.

- Cross-language studies. If a sensory deficit is a primary cause of dyslexia, it should be found across languages. The manifestation of the deficit may vary with language, but this variability should be predictable from normative development. For example, auditory deficits may affect speed rather than accuracy in phoneme awareness tasks in consistent alphabetic orthographies. 
- Testing effects on other cognitive systems. Any putative sensory cause of dyslexia should have predictable and hypothesis-driven effects on other aspects of cognitive development. For example, an auditory processing deficiency should also affect music cognition. 
Box 2

\section{Phonological awareness and the oscillatory hierarchy}

Linguists identify various levels of phonology (see table). The infant brain is sensitive to many of these levels, but reflective awareness of phonology develops more slowly. Measured by the ability to identify and manipulate phonological units, the development of phonological awareness follows a hierarchical sequence in all languages so far studied, and shows a causal relationship with literacy ${ }^{1}$. Reflective awareness of phonemes is not the natural end-point of development, but depends on direct tuition and alphabetic learning (including learning to spell). Illiterate adults ${ }^{102}$ and Chinese adults who have learned to read logographically ${ }^{108}$ (matching characters to meaning) show very poor phoneme awareness. Although infants can make categorical phonemic distinctions, grouping acoustically-distinct sounds (called allophones) together and treating them as the same phoneme, the sounds reflected by the alphabet are an abstraction from the acoustic signal. For example, English spelling convention uses the letter P to represent the second phoneme in words like SPIN and SPOON, and the consonant $\mathrm{T}$ to represent the first phoneme in words like TRACK and TRAY, even though the acoustic sounds are closer to " $b$ " and "ch" 109 . Indeed, beginning spellers misrepresent these sounds, writing the acoustically accurate 'SBN' or 'CHRAK'. Alphabetic learning has lasting effects on the brain: adult oral language processing is affected by spelling knowledge ${ }^{110}$, whereas pre-reading children do not show orthographic effects during oral language judgements ${ }^{111}$.

Recent theories of speech processing based on cortical oscillations identify an oscillatory hierarchy that approximately parallels the phonological hierarchy summarised here. The oscillatory hierarchy underpins the neural encoding of speech ${ }^{112}$, and may provide an acoustic corollary of phonological units. Newer linguistic theories, such as those that propose "rich phonology", argue that representations for words are stored in continuous time as highdimensional spectro-temporal auditory patterns ${ }^{113}$. According to such accounts, phonological awareness is an emergent property of acoustic structure ${ }^{80}$. By contrast, traditional linguistic theories assumed that neural phonological representations involved sequential collections of phonemes "akin to a pronouncing dictionary" ${ }^{114}$. Newer perspectives suggest that the preliterate brain may code language quite differently, prioritising speech rhythm ${ }^{78,80}$. Clearly, the field is wide open for a principled application of the oscillatory hierarchy to the phonological "deficit" in dyslexia, across languages. Phonological awareness of the highest levels in the hierarchy shown here (phrasal and syllable stress, which are rarely marked in orthographies) have been particularly under-studied. 


\begin{tabular}{|l|l|l|l|}
\hline Phonological Level & $\begin{array}{l}\text { Oscillatory } \\
\text { Frequency } \\
\text { (EEG band) }\end{array}$ & Example/s & $\begin{array}{l}\text { Age at which reflective } \\
\text { awareness develops }\end{array}$ \\
\hline Intonational Phrase & $\begin{array}{l}\sim 1 \mathrm{~Hz} \text { and } \\
\text { lower }\end{array}$ & $\begin{array}{l}\text { Who's a pretty } \\
\text { boy then? }\end{array}$ & Not yet ascertained \\
\hline Stressed syllable & $\begin{array}{l}\sim 2 \mathrm{~Hz} \\
\text { (delta) }\end{array}$ & $\begin{array}{l}\text { PE-ter PI-per } \\
\text { PICKED a } \\
\text { PECK of PICK- } \\
\text { led PEPP-ers }\end{array}$ & Not yet ascertained \\
\hline Syllable & $\begin{array}{l}\sim 5 \mathrm{~Hz} \\
\text { (theta) }\end{array}$ & $\begin{array}{l}\text { an }-\mathrm{i}-\mathrm{mal} \\
\text { wig }- \text { wam }\end{array}$ & $2-3$ years \\
\hline Onset-rime & $\begin{array}{l}\sim \text { cued by } \\
\text { rising theta } \\
\text { slope }\end{array}$ & $\begin{array}{l}\mathrm{c}-\text { at } \\
\text { str }- \text { eam } \\
\text { cl - amp }\end{array}$ & $3-4$ years \\
\hline Phoneme & $\begin{array}{l}\sim 35 \mathrm{~Hz} \\
\text { (gamma) }\end{array}$ & c-l-a - m - p & With alphabetic tuition \\
\hline
\end{tabular}


Figure Captions.

\section{Coherent motion detection and visual area 5 (V5) activation in dyslexia.}

As motion is detected by the magnocellular system, coherent motion detection has been the key task used in studies of magnocellular deficits. Depressed neural activity in area V5/MT during task performance has been used as the biological marker of such deficits. A| In the coherent motion task, the child has to detect the direction of motion (left panel) in a dense array of moving dots. For scanning studies, the usual control display is static dots (right panel). B In a study providing phonological training ${ }^{32}$, it was demonstrated that activity in right V5/MT (left panel) during a coherent motion task showed a significant increase after the intervention period (right panel), that was not seen in the control periods. As a significant change in activity (indicated by an asterisk) followed a phonological intervention, this study suggests that the differential performance of dyslexic children in coherent motion tasks is mediated through reading. Part a courtesy of Guinevere Eden, Georgetown University, Washington D.C., USA. Part b modified with permission from ${ }^{32}$.

\section{Performance in the visual span task in dyslexia}

a| The visual span task usually tests visual short-term memory capacity using an array of 5 letters or digits (left panel), but has also been tested with colours and symbols (right panel). The child either reports the whole array or the letter/symbol at a cued location. $b$ The performance of children and adults with dyslexia on this task in CA-match studies is reliably impaired (left panel). However, when symbols or coloured dots are used instead of letters/digits, no reduction in visual span capacity is found (right panel) ${ }^{41}$. Children with dyslexia perform at equivalent levels to age-matched children. This makes it unlikely that the ability to process multiple visual elements in parallel is a primary impairment causing developmental dyslexia. Figure modified with permission from refs 41,45 .

\section{Visuo-spatial cueing tasks.}

A range of Posner cueing tasks have been used in studies of the visual spatial attention deficit in dyslexia, 4 examples of which are shown here. Typically a target's future location is cued briefly, and the dependent measure is whether this spatial cue facilitates subsequent detection of the target. Some studies require the participant to respond to a target presented to the left and right visual fields and compare the reaction time when a valid cue is presented ahead of the target from the reaction time when an invalid cue is used $\left(a, b^{48,49}\right)$. By varying the length of the interval between cue and target $(350 \mathrm{~ms}$ in a and 100 or $250 \mathrm{~ms}$ in $\mathrm{b}$ ), these studies claim to demonstrate sluggish spatial attention shifting in dyslexic individuals (that is, the longer interval is required for successful cue utilisation or the cue is not utilised successfully even with a long interval). Other studies $(\mathrm{c}, \mathrm{d})$ require the cue to be selected from a multiple-element array of targets (ovals in c and false font in $\mathrm{d}^{52,53}$ ) using different temporal intervals between cue and target and using masking in some experimental conditions. The dependent measure is the accuracy of target detection when valid cues are supplied. The variability of paradigm, dependent measure and cue-target asynchronies makes it difficult to extract a consistent 
developmental picture of the exact nature of dyslexic impairment. Part a modified with permission from ref 48. Part b modified, with permission from ref 49. Part c modified, with permission from ref 52. Part d modified, with permission from ref 53.

\section{Examples of linguistic information assessed by auditory theories.}

Schematic depiction of the linguistic information that it is claimed is affected by nonspeech auditory processing deficits in the detection of rapid frequency changes ${ }^{59}$ and the detection of amplitude modulations below $10 \mathrm{~Hz}^{78}$.Aal Image of the speech spectogram shows how the frequency of the speech signal changes over time. Ab,Ac| Higher magnification view of the changes in frequency as the syllables "ba" and "da" (in the words 'bar' and 'dark') are spoken. The rapid rises and falls in frequency visible in the first $40 \mathrm{~ms}$ of the stimuli distinguish the different syllables, which are indicated by white lines ${ }^{59} . \mathrm{Ba}, \mathrm{Bb} \mid$ Changes in amplitude modulation at three different linguistically-relevant temporal rates (stressed syllable, syllable and phonetic rate) are shown for the 4-syllable words "comfortable" (primary stress on first syllable) and "debateable" (primary stress on second syllable; raw acoustic signal shown in black ${ }^{115}$ ) spoken in real time. The whole modulation spectrum of the envelope is shown ( $\mathrm{z}$ axis). The figure shows clearly that information in the lower spectral frequencies (foreground of each rate, $<\sim 700 \mathrm{~Hz}$ ) carries the most information about syllabic patterning. The Stress band shows highest amplitude modulation for the stressed syllable in each case, whereas the Syllable band shows an amplitude peak for each individual syllable. Phase alignment of the amplitude peaks at the Stress and Syllable rates contributes to prosodic prominence ${ }^{80,81,115}$ (indicated by arrows). Panel A reproduced with permission from $\mathrm{REF}^{59}$. Panel B reproduced with permission from $\mathrm{REF}^{115}$. 


\section{Bibliography}

1. Ziegler, J.C. \& Goswami, U. Reading acquisition, developmental dyslexia, and skilled reading across languages: A psycholinguistic grain size theory. Psychol Bull. 131, 3-29 (2005).

2. Foresight Mental Capital and Wellbeing Project: Final Project Report. The Government Office for Science, London, 2008.

3. Goswami, U., Power, A.J., Lallier, M. \& Facoetti, A. Oscillatory 'temporal sampling' and developmental dyslexia: Towards an over-arching theoretical framework. Front. Hum. Neurosci. (in press).

4. Giraud, A-L., \& Ramus, F. Neurogenetics and auditory processing in developmental dyslexia. Curr. Opin. Neurobiol. 22, 37-42. (2013).

5. Carrion-Castillo, A., Franke, B., \& Fisher, S.E. Molecular genetics of dyslexia: An overview. Dyslexia. 19, 214-240. (2013).

6. Clark, K., et al. Neuroanatomical precursors of dyslexia identified from pre-reading through age 11. Brain (in press).

7. Goswami, U. Why theories about developmental dyslexia require developmental designs. Trends Cogn. Sci. 7, 534-540 (2003).

8. Frost, R. Towards a universal model of reading. Behav. Brain Sci. 35, 263-279 (2012).

9. Seymour, P. H. K., Aro, M. \& Erskine, J. M. Foundation literacy acquisition in European orthographies. Brit. J. Psychol. 94, 143-174 (2003).

10. Ziegler, J. C. et al. Orthographic depth and its impact on universal predictors of reading: A cross-language investigation. Psychol. Sci. 21, 4, 551-559 (2010).

11. Vellutino, F. R. Dyslexia. Cambridge, MA: MIT Press (1979).

12. Huang H. S. \& Hanley J. R. (1994). Phonological Awareness and Visual Skills in learning to Read Chinese and English. Cognition. 54,_73-98 (1994).

13. McBride-Chang, C., et al. Visual spatial skill: A consequence of learning to read? .J Exp. Child Psychol. 109, 256-262 (2011).

14. Programme for International Student Assessment. OECD (2009).

15. Snowling, M. J. Dyslexia (2nd ed.). Blackwell, Oxford, UK- (2000).

16. Swan, D. \& Goswami, U. Picture Naming Deficits in Developmental Dyslexia: The Phonological Representations Hypothesis. Brain Lang. 56, 334-353 (1997).

17. Kuhl, P. K. Early language acquisition: Cracking the speech code. Nat. Rev. Neurosci. 5, 831-843 (2004).

18. Stanovich, K.E. Matthew effects in reading: Some consequences of individual differences in the acquisition of literacy. Read Res. Quart, 21, 360-407 (1986).

19. Mitchell, T.V. \& Neville, H. J. Asynchronies in the development of electrophysiological responses to motion and colour. J. Cognitive Neurosci. 16, 8, 1363-1374 (2004).

20. Stein, J. \& Walsh, V. To see but not to read: The magnocellular theory of dyslexia. Trends Neurosci. 20, 147-152 (1997).

21. Lovegrove, W. A., Bowling, A., Badcock, D. \& Blackwood, M. Specific reading disability: Differences in contrast sensitivity as a function of spatial frequency. Science, 210, 439-440 (1980).

22. Livingstone, M. S., Rosen, G. D., Drislane, F. W. \& Galaburda, A. M. Physiological and anatomical evidence for a magnocelluar deficit in developmental dyslexia. PNAS, $\mathbf{8 8}$, 7943-7947 (1991).

23. Cornelisson, P., Richardson, A., Mason, A., Fowler, S. \& Stein, J. Contrast sensitivity and coherent motion detection measured at photopic luminance levels in dyslexics and controls. Vision Res. 35, 1483-1494 (1995).

24. Witton, C. et al. Sensitivity to dynamic auditory and visual stimuli predicts nonword reading ability in both dyslexic and normal readers. Curr. Biol. 8, 791-797 (1998). 
25. Kevan, A. \& Pammer, K. Predicting early reading skills from pre-reading measures of dorsal stream functioning. Neuropsychologia, 47, 3174-3181 (2009).

26. Dehaene, S. et al. Why do children make mirror errors in reading? Neural correlates of mirror invariance in the visual word form area. Neuroimage, 49, 1837-48 (2010).

27. Eden, G. et al. Abnormal processing of visual motion in dyslexia revealed by functional neuroimaging. Neuron. 21, 279-282 (1996).

28. Gori, S., Cecchini, P., Bigoni, A., Molteni, M., \& Facoetti, A. Magnocellular-dorsal pathway and sub-lexical route in developmental dyslexia. Front. Hum. Neurosci. 8:460 (2014).

29. Hutzler, F., Kronbichler, M., Jacobs, A. M. \& Wimmer, H. Perhaps correlational but not causal: No effect of dyslexic readers' magnocellular system on their eye movements during reading. Neuropsychologia. 44, 637-648 (2006).

30. Boets, B., Wouters, J., van Wieringen, A., De Smedt, B. \& Ghesquiere, P. Modelling relations between sensory processing, speech perception, orthographic and phonological ability, and literacy achievement. Brain Lang. 106, 29-40 (2008).

31. Sperling, A. J., Lu, Z. L., Manis, F. R. \& Seidenberg, M. S. Deficits in perceptual noise exclusion in developmental dyslexia. Nat. Neurosci. 8, 862-3 (2006).

32. Olulade, O. A., Napoliello, E. M. \& Eden, G. F. Abnormal visual motion processing is not a cause of dyslexia. Neuron. 79, 180-190 (2013).

33. Brand, R.J., Baldwin, D.A. \& Ashburn, L.A. Evidence for motionese: modifications in mothers' infant-directed action. Dev. Sci. 5, 72-83 (2002).

34. Amitay, S., Ben-Yehudah, G., Banai, K. \& Ahissar, M. Disabled readers suffer from visual and auditory impairments but not from a specific magnocellular deficit. Brain, 125, 2272-2285 (2002).

35. Atkinson, J. et al. A specific deficit of dorsal stream function in Williams' syndrome. Neuroreport. 8, 1919-1922 (1997).

36. Bosse, M. L., Tainturier, M. J. \& Valdois, S. Developmental dyslexia: The visual attention span deficit hypothesis. Cognition. 104, 198-230 (2007).

37. Bosse, M. L. \& Valdois, S. Influence of the visual attention span on child reading performance: A cross-sectional study. J. Read Res. 32, 230-253 (2009).

38. Bosse, M.L. \& Valdois, S. Patterns of developmental dyslexia according to a multi-trace memory model of reading. Curr. Psychol. Lett. 10, 1. (2003).

39. Spinelli, D., De Luca, M., Judica, A. \& Zocolotti, P. Crowding effects on word identification in developmental dyslexia. Cortex, 38, 179-200 (2002).

40. Zoccolotti, P., De Luca, M., Di Filippo, G. \& Martelli, M. Markers of surface developmental dyslexia in a language (Italian) with high grapheme-phoneme correspondence. Appl. Psycholinguist. 20, 191-216 (1999).

41. Ziegler, J. C. et al. Rapid processing of letters, digits and symbols: What purely visualattentional deficit in developmental dyslexia? Dev. Sci. 13, F8-F14 (2010).

42. Maurer, U. et al. Impaired tuning of a fast occipito-temporal response for print in dyslexic children learning to read. Brain. 130, 3200-3210 (2007).

43. Blau, V. et al. Deviant processing of letters and speech sounds as proximate cause of reading failure: A functional magnetic resonance imaging study of dyslexic children. Brain. 133, 868-879 (2010).

44. Fernandes, T., Vale, A. P., Martins, B., Morais, J. \& Kolinsky, R.The deficit of letter processing in developmental dyslexia: Combining evidence from dyslexics, typical readers and illiterate adults. Dev. Sci. 17, 1, 125-141 (2014).

45. Valdois, S., Lassus-Sangosse, D. \& Lobier, M. Impaired letter string processing in developmental dyslexia: What visual-to-phonology code disorder? Dyslexia. 18, 77-93 (2012). 
46. Zorzi, M. et al. Extra-large letter spacing improves reading in dyslexia. PNAS. 109, 11455-11459 (2012).

47. Facoetti, A. et al. The time course of attentional focusing in dyslexic and normallyreading children. Brain Cognition. 53, 181-184 (2003).

48. Facoetti, A. et al. The relationship between visuo-spatial attention and nonword reading in developmental dyslexia. Cognitive Neuropsych. 23, 6, 841-855 (2006).

49. Facoetti, A. et al. Multisensory spatial attention deficits are predictive of phonological decoding skills in developmental dyslexia. J. Cognitive Neurosci. 22, 1011-1025 (2010).

50. Ross-Sheehy,S., Oakes, L. M. \& Luck, S. J. Exogenous attention influences visual short-term memory in infants. Dev. Sci. 14, 490-501 (2011).

51. Hari, R., Renvall, H. \& Tanskanen, T. Left mini-neglect in dyslexic adults. Brain. 124, 1373-80 (2001).

52. Franceschini, S. et al. A causal link between visual spatial attention and reading acquisition. Curr. Biol. 22, 814-819 (2012).

53. Franceschini, S. et al. Action video games make dyslexic children read better. Curr. Biol. 23, 462-466 (2013).

54. Hawelka, S. \& Wimmer, H. Impaired visual processing of multi-element arrays is associated with increased number of eye movements in dyslexic reading. Vision Res. 45, 855-863 (2005).

55. Vidyasagar, T. R. \& Pammer, K. Dyslexia: a deficit in visuo-spatial attention, not in phonological processing. Trends Cogn. Sci. 14, 57-63 (2010).

56. Mehler, J., Jusczyk, P., Lambertz, G., Halsted, N., Bertoncini, J. \& Amiel-Tison, C. A precursor of language acquisition in young infants. Cognition. 29, 143-178 (1988).

57. Telkemeyer, S. et al. Acoustic processing of temporally-modulated sounds in infants: Evidence from a combined NIRS and EEG study. Front. Psychol. 2, 62 (2011).

58. Nittrouer, S. \& Lowenstein, J. H. Perceptual organization of speech signals by children with and without dyslexia. Res. Dev. Disabil. 34, 2304-2325 (2013).

59. Tallal, P. Improving language and literacy is a matter of time. Nat. Rev. Neurosci. 5, 721-728 (2004).

60. Tallal, P. \& Piercy, M. Defects of non-verbal auditory perception in children with developmental aphasia. Nature, 241, 468-469 (1973).

61. Tallal, P. \& Piercy, M. Developmental aphasia: Rate of auditory processing and selective impairment of consonant perception. Neuropsychologia, 12, 83-93 (1974).

62. Tallal, P. Auditory temporal perception, phonics, and reading disabilities in children. Brain Lang. 9, 182-198 (1980).

63. Marshall, C. M., Snowling, M. J. \& Bailey, P. J. Rapid auditory processing and phonological ability in normal readers and readers with dyslexia. J. Speech Lang. Hear. Res. 44, 925-40 (2001).

64. McArthur, G. M. \& Bishop, D. V. M. Auditory perceptual processing in people with reading and oral language impairments: Current issues and recommendations. Dyslexia. 7, 150-170 (2001).

65. Studdert-Kennedy, M. \& Mody, M. Auditory temporal perception deficits in the reading-impaired: A critical review of the evidence. Psychon. B. Rev. 2, 508-514 (1995).

66. McAnally, K. I., Hansen, P. C., Cornelissen, P. L. \& Stein, J. F. Effect of time and frequency manipulation on syllable perception in developmental dyslexics. J. Speech Lang. Hear. Res. 40, 912-924 (1997).

67. Temple, E. et al. Neural deficits in children with dyslexia ameliorated by behavioural remediation: Evidence from functional MRI. PNAS. 100, 5, 2860-2865. (2003). 
68. Gaab, N., Gabrieli, J. E., Deutsch, G. K., Tallal, P. \& Temple, E. Neural correlates of rapid auditory processing are disrupted in children with developmental dyslexia and ameliorated with training: An fMRI study. Restor. Neurol.Neuros. 25, 295-310 (2007).

69. Raschle, N., Chang, M. \& Gaab, N. Structural brain alterations associated with dyslexia predate reading onset. Neuroimage, 57, 742-9 (2011).

70. Choudray, N. \& Benasich, A.A. Maturation of evoked auditory potentials from 6 to 48 months: Prediction to 3- and 4-year language and cognitive abilities. Clin. Neurophysiol. 122, 320-38 (2011).

71. Boets, B., et al. Preschool impairments in auditory processing and speech perception uniquely predict future readings problems. Res. Dev. Disabil. 32, 560-570 (2011).

72. Goswami, U., Fosker, T., Huss, M., Mead, N. \& Szűcs, D. Rise time and formant transition duration in the discrimination of speech sounds: The Ba-Wa distinction in developmental dyslexia. Dev. Sci. 14, 34-43 (2011).

73. Lehongre, K., Ramus, F., Villiermet, N., Schwartz, D. \& Giraud A-L.. Altered lowgamma sampling in auditory cortex accounts for the three main facets of dyslexia. Neuron. 72, 1080-1090 (2011).

74. Boets, B. et al. Intact but less accessible phonetic representations in adults with dyslexia. Science. 342, 1251-4 (2013).

75. Serniclaes, W., van Heghe, S., Mousty, P., Carre, R., \& Sprenger-Charolles, L. Allophonic mode of speech perception in dyslexia. J. Exp. Child Psychol. 87, 336-61 (2004).

76. Bogliotti, C., Serniclaes, W., Messaoud-Galusi, S. \& Sprenger-Charolles, L. Discrimination of speech sounds by children with dyslexia: Comparisons with chronological age and reading level controls. J. Exp. Child Psychol. 101, 137-155 (2008).

77. Goswami, U. et al. Amplitude envelope onsets and developmental dyslexia: A new hypothesis. PNAS. 99, 10911-10916 (2002).

78. Goswami, U. A temporal sampling framework for developmental dyslexia. Trends Cogn. Sci., 15, 3-10 (2011).

79. Leong, V. Prosodic rhythm in the speech amplitude envelope: Amplitude modulation phase hierarchies (AMPHs) and AMPH models. (Doctoral dissertation, University of Cambridge, 2012).

80. Leong, V. \& Goswami, U. Assessment of rhythmic entrainment at multiple timescales in dyslexia: Evidence for disruption to syllable timing. Hear. Res. 308, 141-161. (2014).

81. Leong, V., \& Goswami, U. Impaired extraction of speech rhythm from temporal modulation patterns in speech in developmental dyslexia. Front. Hum. Neurosci. 8, 96.

82. Goswami, U., Wang, H. -L. S., Cruz, A., Fosker, T., Mead, N. \& Huss, M. Languageuniversal sensory deficits in developmental dyslexia: English, Spanish, and Chinese. $J$ Cognitive Neurosci. 23, 325-337 (2011).

83. Muneaux, M., Ziegler, J. C., Truc, C., Thomson, J. \& Goswami, U. Deficits in beat perception and dyslexia: Evidence from French. NeuroReport. 15, 1255-1259 (2004).

84. Surányi, Z. et al. Sensitivity to rhythmic parameters in dyslexic children: A comparison of Hungarian and English. Read. Writ. 22, 41-56 (2009).

85. Hämäläinen, J. A., Leppänen, P. H. T., Guttorm, T. K. \& Lyytinen, H. Event-related potentials to pitch and rise time change in children with reading disabilities and typically reading children. Clin. Neurophysiol. 119, 100-115 (2008).

86. Poelmans, H. et al. Reduced sensitivity to slow-rate dynamic auditory information in children with dyslexia. Res. Dev. Disabil. 32, 2810-2819 (2011). 
87. Papadopoulos, T. C., Georgiou, G. K. \& Parrila, R. K. Low-level deficits in beat perception: Neither necessary nor sufficient for explaining developmental dyslexia in a consistent orthography. Res. Dev. Disabil. 33, 1841-56. (2012).

88. Goswami U. et al. Impaired perception of syllable stress in children with dyslexia: A longitudinal study. J. Mem. Lang. 69, 1, 1-17 (2013).

89. Kitzen, K.R. Prosodic sensitivity, morphological ability, and reading ability in young adults with and without childhood histories of reading difficulty. Dissertation Abstracts International. 62 (02), 0460A. (2001).

90. Thomson, J. \& Goswami, U. Rhythmic processing in children with developmental dyslexia: Auditory and motor rhythms link to reading and spelling. J. Physiol. Paris. 102, 120-129. (2008).

91. Goswami, U., Huss, M., Mead, N., Fosker, T. \& Verney, J. P. Perception of Patterns of Musical Beat Distribution in Phonological Developmental Dyslexia: Significant Longitudinal Relations with Word Reading and Reading Comprehension. Cortex. 49, 5, 1363-1376 (2013).

92. Huss, M., Verney, J. P., Fosker, T., Mead, N. \& Goswami, U. Music, rhythm, rise time perception and developmental dyslexia: Perception of musical meter predicts reading and phonology. Cortex. 47, 674-89 (2011).

93. Corriveau, K. H., Goswami, U. \& Thomson, J. M. Auditory processing and early literacy skills in a preschool and kindergarten population. J. Learn. Disabil. 43, 369-382 (2010).

94. Guttorm, T. K., Leppanen, P. H., Hämäläinen, J. A., Eklund, K. M. \& Lyytinen, H. J. Newborn event-related potentials predict poorer pre-reading skills in children at risk for dyslexia. J. Learn. Disabil. 43, 391-401. (2010).

95. Leppanen, P. H. T. et al. Newborn brain event-related potentials revealing atypical processing of sound frequency and the subsequent association with later literacy skills in children with familial dyslexia. Cortex. 46, 1362-1376 (2010).

96. Van Zuijen, T.L., Plakas, A., Maassen, B.A.M., Maurits, N.M., \& van der Leij, A. Infants ERPs separate children at risk of dyslexia who become good readers from those who become poor readers. Dev. Sci. 16, 554-63 (2013).

97. Smith, A.B., Lambrecht-Smith, S., Locke, J.L., \& Bennett, J. A longitudinal study of speech timing in young children later found to have a reading disability. J. Speech Lang. Hear. Res. 51, 1300-14. (2008).

98. Thomson, J., Leong, V., \& Goswami, U. Auditory processing interventions and developmental dyslexia: A comparison of phonemic and rhythmic approaches. Read. Writ. 26, 139-161. (2013).

99. Bhide, A., Power, A. J. \& Goswami, U. A rhythmic musical intervention for poor readers: A comparison of efficacy with a letter-based intervention. Mind Brain Educ. 7, 2, 1-12 (2013).

100. Stefanics, G. et al . Auditory sensory deficits in developmental dyslexia, a longitudinal study. Neuroimag., 57, 723-32 (2011).

101. Power, A. J., Mead, N., Barnes, L. \& Goswami, U. Neural entrainment to rhythmic speech in children with dyslexia. Front. Hum. Neurosci. 7, 777 (2013).

102. De Santos Loureiro et al. Degree of illiteracy and phonological and metaphonological skills in unschooled adults. Brain Lang. 89, 499-502 (2004).

103. Schneps, M. H. et al. Shorter lines facilitate reading in those who struggle. PLOS ONE. 8, 8, e71161 (2013).

104. Bradley, L. \& Bryant, P. E. Difficulties in auditory organisation as a possible cause of reading backwardness. Nature. 271, 746-747 (1978). 
105. Megnin-Viggars, O. \& Goswami, U. Audiovisual perception of noise vocoded speech in dyslexic and non-dyslexic adults: The role of low-frequency visual modulations. Brain Lang. 124, 2, 165-73 (2013).

106. Vidyasagar, T. R. Reading into neuronal oscillations in the visual system: Implications for developmental dyslexia. Front. Hum. Neurosci. 7, 811 (2013).

107. Pammer, K. Temporal sampling in vision and implications for dyslexia. Front. Hum. Neurosci. 7, 933 (2013).

108. Read, C., Zhang, Y-F., Nie, H-Y. \& Ding, B-Q. The ability to manipulate speech sounds depends on knowing alphabetic writing. Cognition. 24, 31-44 (1986).

109. Read, C. Children's creative spelling. Routledge Kegan Paul, London, UK (1986).

110. Ziegler, J. C. \& Ferrand, L. Orthography shapes the perception of speech: The consistency effect in auditory word recognition. Psychon. B. \& Rev. 5, 4, 683-689 (1998).

111. Goswami, U., Ziegler, J. C. \& Richardson, U. The effects of spelling consistency on phonological awareness: A comparison of English and German. .J. Exp. Child Psychol. 92, 4, 345-365 (2005).

112. Giraud, A. L., \& Poeppel, D. Cortical oscillations and speech processing: emerging computational principles and operations. Nat. Neurosci. 15, 4, 511-517 (2012).

113. Port, R. How are words stored in memory? Beyond phones and phonemes. New Ideas Psychol. 25, 143-170 (2007).

114. Greenberg, S. Speaking in shorthand- A syllable-centric perspective for understanding pronunciation variation. Speech Commun. 29, 159-176 (1999).

115. Goswami, U., \& Leong, L. Speech rhythm and temporal structure: Converging perspectives? Lab. Phonol. 4, 1, 67-92. 


\section{Glossary}

Cortical oscillations: The synchronous firing of neurons in networks of various sizes in different areas of the cortex, producing rhythmic patterns of activity. These oscillatory rhythms have endogenous temporal rates, and can phase-reset their activity to synchronise firing with incoming sensory information, thereby contributing to the processing of the input.

Formant transitions: formants are concentrations of acoustic energy within a narrow frequency band in the speech signal. They change rapidly (within $40 \mathrm{~ms}$ ) as the speaker transitions from a consonant to a vowel, providing important cues to phonetic identity.

Frequency doubling illusion: an illusion that depends on the spatial and temporal frequency of a flickering sinusoid grating (a pattern of lighter and darker bars). When a grating with a spatial frequency of $0.1-0.4$ cycles/degree flickers faster than $15 \mathrm{~Hz}$, the viewer sees a grating with much narrower lines (i.e., the physical spatial frequency appears to double).

Graphemes: letters or clusters of letters that correspond to a single speech sound (phoneme), such as $\mathrm{PH}$ or $\mathrm{CH}$.

Logographic: direct recognition of the meaning of a symbol without requiring recoding to sound. English logographs include $£, \%$ and $>$.

Magnocellular system: one of two major pathways in the visual system, with larger cell bodies than the other (parvocellular) system. Magnocells respond optimally to motion and to visual stimuli that reverse contrast (areas of light versus dark) at lower spatial frequencies and at higher temporal frequencies. Responses are transient and the system is colour blind.

Mental lexicon: the brain's mental dictionary, containing information about the meaning, pronunciation and grammatical status of words in the spoken language.

Morphology: information concerning how words are formed in language, comprising knowledge of specific patterns or regularities in the way that words are created from smaller linguistic units such as root meanings and grammatical markers.

Onset-rime division: when a spoken syllable is divided at the vowel or syllable nucleus, the consonant phoneme/s preceding the vowel are the linguistic onset and the vowel and any subsequent consonant phoneme/s are the linguistic rime, as in s-ee, s-eep, sl-eep, sl-ept.

Orthography: the correct writing system of a language, used here to refer to the chosen symbol-sound correspondence system (e.g., Western alphabet, Cyrillic alphabet, Chinese characters, Devanagari).

Phonemes: the smallest units of sound that change a word's meaning.

Phonology: the inventory of the sound system of a language, comprising knowledge of the sounds themselves and the specific patterns or regularities by which sounds in words can be organised.

Posner cueing tasks: a neuropsychology paradigm for measuring spatial attention by cueing a target's future location and measuring whether this cue facilitates target detection. A Posner task usually includes a contrast between valid and invalid cues (only valid cues indicate the 
future correct location) and endogenous versus exogenous cues (endogenous cues are central to the visual field while exogenous cues are outside the focus of attention or in the periphery).

Saccade: a rapid jerk-like movement of the eyeball which redirects the fovea to a new location in the visual field without a head movement or the conscious awareness of the observer.

SLI: specific language impairment is a developmental disorder of language acquisition that delays the mastery of skills in children who have no hearing loss or other developmental delays.

Syntax: the rules governing how words or other linguistic elements such as phrases are combined to form grammatically correct utterances.

\section{Author Biography}

Usha Goswami FBA is Professor of Cognitive Developmental Neuroscience at the University of Cambridge and Director of the Centre for Neuroscience in Education. After training as a teacher, she began to research the acquisition of reading across languages, proposing a key role for rhyme analogy in English. She then worked on children's analogical reasoning. Her more recent research examines relations between phonology and dyslexia across languages, with a focus on the neural oscillatory underpinnings of speech prosody and rhythm. She is also working on a range of neuroeducational interventions based on poetry and rhythm, plus the software intervention GraphoGame Rime. 